\section{Utilização de serviços odontológicos e fatores associados: um estudo de base populacional no Sul do Brasil}

\author{
Use of dental services and associated factors: \\ a population-based study in southern Brazil
}

\section{Programa de Pós-graduação em Epidemiologia, \\ Universidade Federal de \\ Pelotas, Pelotas, Brasil. \\ 2 Universidade Federal da \\ Grande Dourados, Dourados, \\ Brasil. \\ 3 Programa de Pós-graduação \\ em Saúde Pública, \\ Universidade Federal de \\ Santa Catarina, \\ Florianópolis, Brasil. \\ Correspondência \\ C. S. Araújo \\ Programa de Pós-graduação \\ em Epidemiologia, \\ Universidade Federal de \\ Pelotas. \\ Rua General Osório 257 \\ Pelotas, RS \\ 96020-000, Brasil. \\ araujo.cs@gmail.com}

\section{Abstract}

The aim of this study was to estimate patterns of dental services use in a medium-sized city in southern Brazil. A population-based crosssectional study was conducted in a sample of 4,226 individuals aged 10 years and older. Poisson regression analysis was used to identify associated factors; $50.9 \%$ (95\% CI: 49.3-52.4) of the sample had consulted a dentist in the previous year. Dental care attendance rates among adolescents, adults, and elders were $52.2 \%, 53.6 \%$, and $37.2 \%$, respectively. After adjusting for various confounders, the most important determinants of dental services use were: schooling $\geq 9$ years, high socioeconomic status, and self-reported oral health problems. In addition, adolescents and adults that rated their oral health as good showed 10\% and 40\% higher prevalence dental services use, respectively, when compared with those who rated their oral health as bad. Determining the prevalence of dental services use and users' characteristics can help policymakers plan future measures to make dental care available to population groups with low rates of dental services use.

Dental Health Services; Dental Care; Oral Health
Camila Silveira de Araújo 1 Rosângela da Costa Lima 2 Marco Aurélio Peres 3 Aluísio J. D. Barros 1

\section{Introdução}

No Brasil, existem poucos estudos epidemiológicos de base populacional descrevendo a utilização de serviços odontológicos 1,2. O suplemento de saúde da Pesquisa Nacional por Amostra de Domicílios (PNAD), realizada em 1998 pelo Instituto Brasileiro de Geografia e Estatística (IBGE), englobou dados referentes à morbidade, ao acesso e à utilização dos serviços, dentre outros aspectos relacionados à saúde 3 . Neste estudo, verificou-se que houve uma prevalência de utilização de serviços odontológicos, no último ano, de apenas $33 \%$, e que $18,7 \%$ dos brasileiros nunca haviam consultado um dentista 2,4 . Na PNAD de 2003, o suplemento saúde foi novamente aplicado e revelou pequenos avanços, como redução para 15,9\% do percentual de pessoas que nunca haviam consultado dentista 5 . Esses inquéritos também evidenciaram enorme desigualdade na utilização dos serviços odontológicos. Enquanto 38,5\% das pessoas nunca consultaram com dentista entre os mais pobres, esse fato foi relatado por apenas $4 \%$ do quinto mais rico da população.

Outro estudo epidemiológico nacional de saúde bucal, intitulado projeto SB Brasil e realizado nos anos de 2002-2003 6, apresentou, para a Região Sul, prevalência de consulta, há menos de um ano, de $60,8 \%, 46,6 \%$ e $23,5 \%$ para os grupos de 15-19 anos, 35-44 anos e 65-74 anos, respectivamente. 
Estudos epidemiológicos de base nacional revelam uma crescente melhoria nos padrões de ocorrência de cárie dentária em crianças nas últimas décadas ${ }^{6}$. Entretanto, entre os adultos e idosos, o quadro ainda é muito grave. Por exemplo, mais de $28 \%$ dos adultos não têm nenhum dente funcional em, pelo menos, uma arcada 6 . Apesar de a consulta com dentista não resultar, necessariamente, em diminuição da ocorrência de doenças bucais, a utilização de serviços odontológicos é um direito, mitiga os problemas, alivia a dor e o sofrimento e reabilita estruturas perdidas 7 . A utilização de serviços está associada a diversos fatores, tais como o nível econômico, educacional e social, bem como padrões de cultura e tradição popular 8,9,10, os quais têm sido pesquisados em vários países, sobretudo no Canadá, Estados Unidos, Noruega e Inglaterra 11,12,13. Na maior parte desses países, o uso de serviços odontológicos tende a ser maior nos grupos de maiores renda 9 e escolaridade 14 .

Pesquisa bibliográfica buscando identificar estudos epidemiológicos de base populacional sobre utilização de serviços odontológicos nas bases de dados PubMed, LILACS e Bibliografia Brasileira de Odontologia (BBO), sem restrição ao ano de publicação, encontrou apenas os estudos baseados nas PNAD $1998{ }^{3}$ e $2003{ }^{5}$ e o da cidade de Bambuí, oeste de Minas Gerais, que incluiu apenas idosos 15 .

Em vista da quantidade limitada de informação sobre a utilização dos serviços odontológicos no Brasil, o presente estudo investiga padrões de utilização por faixa etária e determinantes sócioeconômicos, além da auto-avaliação de saúde bucal e presença de problema odontológico.

\section{Métodos}

Este estudo foi realizado em Pelotas, Rio Grande do Sul, Brasil, cidade de, aproximadamente, 320 mil habitantes com 93\% de residentes na área urbana. Consistiu de um estudo transversal, de base populacional, com aplicação de entrevistas em indivíduos a partir de dez anos de idade, moradores da zona urbana. Indivíduos institucionalizados em asilos, hospitais, presídios ou incapacitados de responder ao questionário foram excluídos. O trabalho de campo ocorreu no período de outubro a dezembro de 2005 e foi executado por uma equipe composta por 38 entrevistadoras e 11 supervisores de campo.

O tamanho da amostra foi calculado visando atender tanto o estudo de prevalência como o estudo de associação entre utilização de serviços, o desfecho e as variáveis independentes. Com o objetivo de estimar a prevalência de utili- zação de serviços odontológicos no ano anterior à pesquisa, foram considerados os seguintes parâmetros: prevalência de 35\%, erro de três pontos percentuais e um nível de $95 \%$ de confiança, com acréscimo de $10 \%$ para compensar perdas e recusas, e mais $100 \%$ devido ao efeito de delineamento esperado, decorrente da amostragem por conglomerados. Desse cálculo, resultou uma amostra de 2.130 indivíduos. Para o estudo das associações, o maior tamanho de amostra foi de 1.022 pessoas, utilizando-se probabilidade do erro tipo $\mathrm{I}<5 \%$, poder de $80 \%$, razão entre não expostos e expostos de 2:8, prevalência de utilização de serviços odontológicos de $21 \%$ no grupo não exposto e razão de prevalências de 1,8, após acréscimo de $10 \%$ para perdas e recusas, $15 \%$ para controle de possíveis fatores de confusão e $100 \%$ para efeito de delineamento. Como este estudo foi parte de uma pesquisa mais abrangente, a amostra final excedeu a necessária, totalizando 4.226 indivíduos, aumentando a precisão e o poder do estudo em relação aos cálculos efetuados.

O processo de amostragem foi realizado em dois estágios, baseado em dados do Censo Demográfico de 2000 (IBGE. http://www.ibge.gov.br). Listaram-se todos os setores censitários urbanos elegíveis da cidade (404), em ordem decrescente da média de renda dos chefes de família de cada setor, em uma estratégia de estratificação implícita por nível econômico. Sortearam-se, sistematicamente e com probabilidade proporcional ao tamanho, 120 setores censitários. Posteriormente, uma equipe de auxiliares da pesquisa visitou todos os domicílios de cada setor selecionado, classificando-os quanto à condição de ocupação (se domicílio habitado ou se de comércio). A partir da lista de domicílios particulares habitados, sortearam-se dez domicílios em cada setor, corrigindo-se esse número para variações do número de domicílios enumerados em relação aos dados do censo.

Os moradores dos domicílios selecionados, após serem esclarecidos sobre os objetivos da pesquisa e consentirem em participar, assinando um termo impresso, foram entrevistados por meio de um questionário previamente testado em estudo piloto realizado em setor censitário excluído da amostragem. Os entrevistadores, todos com Ensino Médio completo, eram do sexo feminino e não conheciam os objetivos da pesquisa. Cerca de $10 \%$ das entrevistas foram realizadas duas vezes, utilizando-se um questionário reduzido, por supervisores da pesquisa a fim de se controlar a qualidade da coleta de dados. A entrada de dados foi feita por digitação dupla, com checagem de inconsistências e amplitudes, sendo, para tanto, empregado o programa Epi Info 
versão 6.04 (Centers for Disease Control and Prevention, Atlanta, Estados Unidos).

A variável dependente foi a utilização de serviços odontológicos nos 12 meses anteriores à data da entrevista (sim ou não). As variáveis independentes incluíram sexo (masculino ou feminino); idade (em anos completos); cor da pele auto-referida (branca, preta, amarela, indígena ou parda); nível econômico classificado conforme a Associação Brasileira de Empresas de Pesquisa (ABEP) 16; escolaridade (em anos completos); situação conjugal (com companheiro ou casado, sem companheiro ou solteiro, separado, viúvo); relato de algum problema odontológico nos 12 meses anteriores à data da entrevista (dificuldade para comer ou sentir dor ao tomar líquidos gelados ou quentes; algum dente ou prótese quebrados; cárie ou "buraco" no dente ou prótese; deixar de dormir ou dormir mal por causa dos dentes ou prótese ou algum outro problema na boca ou nos dentes ou prótese); e autopercepção de saúde bucal (ruim/muito ruim, regular ou muito boa/boa). Por tratar-se de grupos pequenos e não passíveis de formar uma categoria específica, os indivíduos de cor da pele amarela foram incorporados aos de cor branca - confi- gurando a categoria "branca e amarela" -; e os indígenas agrupados aos de cor preta e parda, esses três últimos formando a categoria "pretos, pardos e indígenas".

Por entender-se que os determinantes da utilização de serviços odontológicos podem diferir segundo a idade, a amostra foi estratificada, segundo recomendação da Organização Mundial da Saúde (OMS) 17, nas seguintes faixas etárias: 10-19 anos (adolescentes), 20-59 anos (adultos), 60 anos ou mais (idosos).

Calculou-se a prevalência do desfecho, conforme as variáveis independentes, e, a seguir, realizou-se a análise de regressão de Poisson 18 a fim de estimar as razões de prevalência bruta e ajustada e respectivos intervalos de $95 \%$ de confiança (IC95\%).Aanálisemultivariávelseguiuummodelo teórico hierárquico de determinação de utilização de serviços odontológicos (Figura 1). Esse modelo foi baseado no proposto pelo Projeto de Desenvolvimento de Metodologia de Avaliação do Desempenho do Sistema de Saúde Brasileiro (http:// www.proadess.cict.fiocruz.br/index2v.htm, acessado em31/Out/2006), que estabeleceuma cadeia de determinantes hierarquizados que influenciam, de forma distal ou proximal, a utilização

Figura 1

Modelo de análise hierarquizado da utilização de serviços de saúde.

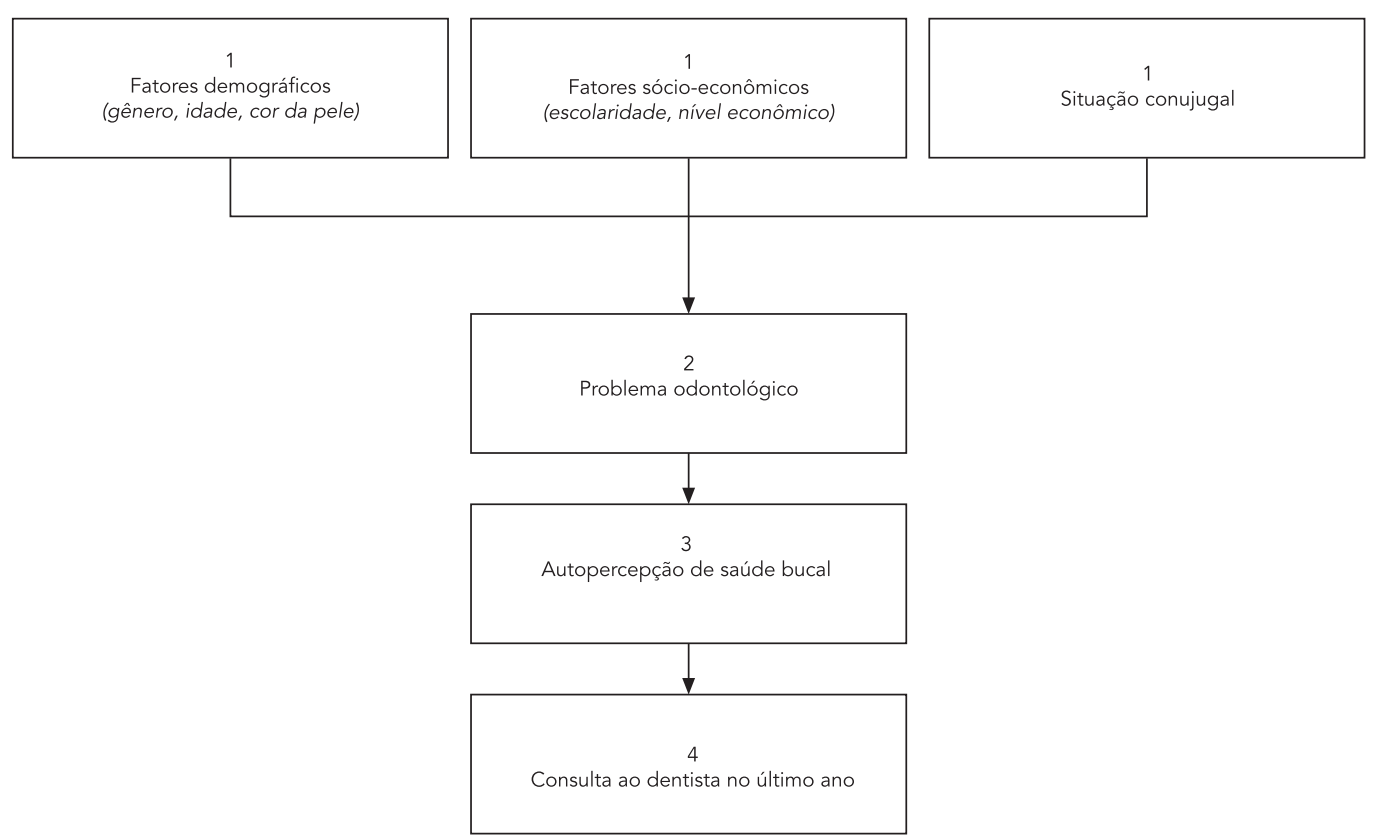


de serviços odontológicos. No nível mais distal de determinação, foram incluídas as características demográficas (gênero, idade e cor da pele), sócio-econômicas (escolaridade e nível econômico) e situação conjugal; no nível intermediário, a variável problema odontológico; e no nível mais proximal, a autopercepção de saúde bucal.

Na modelagem estatística, o efeito de cada variável foi controlado para as variáveis do mesmo nível e nível superior. Foram mantidas, no modelo, as variáveis com valor $\mathrm{p}<0,20$, com a finalidade de se controlar possíveis fatores de confusão. Todas as análises foram realizadas através do programa estatístico Stata 9.0 (Stata Corp., College Station, Estados Unidos), utilizando o prefixo svy: para análise de dados oriundos de amostras complexas, de forma a ajustar as estimativas de variabilidade para a amostragem por conglomerados. Interação entre nível econômico e escolaridade foi testada (teste de Wald para heterogeneidade).

O estudo foi aprovado pela Comissão de Ética e Pesquisa da Faculdade de Medicina, Universidade Federal de Pelotas. Os dados foram coletados após consentimento informado dos entrevistados, sendo-lhes assegurado o sigilo sobre as informações individuais.

\section{Resultados}

Foram entrevistados 3.993 indivíduos, o que equivale a uma taxa de resposta de $94,5 \%$. Desses, $21,5 \%$ tinham idade entre $10-19$ anos; $63,6 \%$, de 20-59 anos; e 14,9\% apresentaram 60 anos ou mais. Os entrevistados tinham, em média, 38 anos de idade (desvio-padrão - DP = 19 anos) e oito anos completos de estudo.

A Tabela 1 apresenta a descrição da amostra estratificada para as idades de 10-19 anos, 20-59 anos e 60 anos ou mais. Em todos os estratos, predominaram mulheres, indivíduos de cor da pele branca, os de nível econômico $\mathrm{C}$ e aqueles com autopercepção de sua saúde bucal classificada como boa ou muito boa.

$\mathrm{O}$ efeito de delineamento amostral para o desfecho sob investigação foi de 3,25 , correspondendo a um coeficiente de correlação intraclasse de 0,06758. A prevalência da utilização de consulta com o dentista nos 12 meses anteriores à entrevista foi de 50,9\% (IC95\%: 49,3-52,4) para a população em geral. Cerca de metade dos adolescentes $(52,2 \%)$ e dos adultos (53,6\%) consultaram nesse período, entretanto, o mesmo não ocorreu com os idosos, pois pouco mais de um terço $(37,2 \%)$ consultou.

Uma parcela de 5,5\% dos entrevistados admitiu nunca ter consultado com dentista, variando de $17,5 \%$ entre adolescentes, $2,4 \%$ nos adultos e $1,5 \%$ nos idosos.

No último ano, mais da metade dos entrevistados $(55,8 \%)$ referiu ter apresentado algum tipo de problema odontológico. Desses, 27,1\% revelaram dificuldade para comer ou sentiu dor ao tomar líquidos gelados ou quentes; $20,4 \%$ fraturaram algum dente ou prótese; $38,8 \%$ referiram cárie dentária; $14,6 \%$ deixaram de dormir ou dormiram mal por causa dos dentes ou prótese; e $13 \%$ tiveram outros problemas na boca, dentes ou prótese. Entretanto, dentre os indivíduos que relataram um ou mais problemas odontológicos, apenas $60,5 \%$ consultaram dentista no último ano. Os indivíduos que relataram ter tido problemas odontológicos alegaram os seguintes motivos para não utilizar os serviços: não precisavam consultar (49,5\%); problemas nos serviços odontológicos $(18,8 \%)$; medo ou preguiça $(17,4 \%)$ e outros motivos (14,3\%).

Ao testar se havia interação entre as variáveis nível econômico e escolaridade, a análise mostrou que o valor de $\mathrm{p}$ ficou muito próximo do limite de significância $(p=0,04)$. Porém, a significância estatística desapareceu quando o termo de interação foi inserido nos modelos multivariáveis para cada faixa etária.

A Tabela 2 apresenta as prevalências de consulta com dentista no último ano, estratificadas por idade e de acordo com variáveis independentes. As mulheres adultas e os adolescentes e adultos de cor branca utilizaram mais os serviços odontológicos. Para todas as faixas etárias estudadas, os indivíduos com melhor nível econômico e maior escolaridade utilizaram mais os serviços odontológicos do que aqueles com pior nível econômico (D/E) e com quatro anos ou menos de escolaridade. Os adolescentes e adultos que consideraram sua saúde bucal como boa ou muito boa consultaram sempre mais do que aqueles com autopercepção regular e ruim ou muito ruim. Além disso, a prevalência de utilização dos serviços odontológicos foi superior para aquelas pessoas que relataram algum tipo de problema bucal.

Na análise bruta (Tabela 2), apenas nos adultos, a situação conjugal e o gênero foram associados com a utilização dos serviços, mostrando que as mulheres e os indivíduos sem companheiro(a) consultam mais que os homens e aqueles(as) com companheiro(a). Além disso, em relação à cor da pele, adolescentes e adultos brancos consultaram, respectivamente, 1,3 e 1,2 vez mais do que os pardos, pretos e indígenas. Após o ajuste (Tabela 3), percebeu-se que as mulheres, na faixa etária de 20-59 anos, consultam $20 \%$ mais do que os homens, entretanto, a cor da pele e a situação conjugal deixaram de se associar com a utilização de serviços odontológicos. 
Descrição da amostra ( $n=3.993$ ) segundo variáveis demográficas, sócio-econômicas e de saúde bucal, estratificada segundo faixas etárias. Pelotas, Rio Grande do Sul, Brasil, 2006.

\begin{tabular}{|c|c|c|c|c|c|c|}
\hline \multirow[t]{3}{*}{ Variável } & \multicolumn{6}{|c|}{ Faixa etária (anos) } \\
\hline & \multicolumn{2}{|c|}{$10-19$} & \multicolumn{2}{|c|}{$20-59$} & \multicolumn{2}{|c|}{$\geq 60$} \\
\hline & $\%$ & $\mathbf{n}$ & $\%$ & $\mathbf{n}$ & $\%$ & $\mathbf{n}$ \\
\hline \multicolumn{7}{|l|}{ Gênero } \\
\hline Masculino & 48,0 & 411 & 45,0 & 1.143 & 39,4 & 235 \\
\hline Feminino & 52,0 & 446 & 55,0 & 1.396 & 60,6 & 362 \\
\hline \multicolumn{7}{|l|}{ Cor da pele } \\
\hline Branca e amarela & 77,9 & 668 & 81,2 & 2.062 & 85,3 & 509 \\
\hline Preta, parda e indígena & 22,1 & 189 & 18,8 & 477 & 14,7 & 88 \\
\hline \multicolumn{7}{|l|}{ Nível econômico * } \\
\hline$D-E$ & 36,3 & 309 & 25,5 & 643 & 32,3 & 192 \\
\hline C & 38,3 & 326 & 42,7 & 1.077 & 36,6 & 218 \\
\hline$A-B$ & 25,4 & 216 & 31,8 & 800 & 31,1 & 185 \\
\hline \multicolumn{7}{|l|}{ Escolaridade (anos) } \\
\hline $0-4$ & 34,5 & 292 & 15,9 & 391 & 42,5 & 212 \\
\hline $5-8$ & 46,6 & 395 & 35,1 & 862 & 28,7 & 143 \\
\hline$\geq 9$ & 18,9 & 160 & 49,0 & 1.202 & 28,9 & 144 \\
\hline \multicolumn{7}{|l|}{ Situação conjugal } \\
\hline Com companheiro(a) & 4,2 & 36 & 64,0 & 1.626 & 57,3 & 342 \\
\hline Sem companheiro(a) & 95,8 & 821 & 36,0 & 913 & 42,7 & 255 \\
\hline \multicolumn{7}{|l|}{ Problema odontológico } \\
\hline Não & 38,2 & 327 & 40,2 & 1.020 & 69,8 & 417 \\
\hline Sim & 61,8 & 530 & 59,8 & 1.519 & 30,2 & 180 \\
\hline \multicolumn{7}{|c|}{ Autopercepção de saúde bucal } \\
\hline Ruim/Muito ruim & 4,4 & 38 & 10,0 & 255 & 7,9 & 47 \\
\hline Regular & 26,7 & 229 & 31,9 & 810 & 25,8 & 154 \\
\hline Boa/Muito boa & 68,8 & 590 & 58,1 & 1.474 & 66,3 & 396 \\
\hline Total & 21,5 & 857 & 63,6 & 2.539 & 14,9 & 597 \\
\hline
\end{tabular}

* Classificação econômica da Associação Brasileira de Empresas de Pesquisa 16.

Adolescentes e adultos com melhor saúde bucal (autopercepção) consultaram mais do que aqueles com saúde ruim ou muito ruim. Esse dado confirmou-se na análise ajustada (Tabela 3). Ademais, os indivíduos que perceberam algum problema odontológico utilizaram de $40 \%$ (adolescentes) a $100 \%$ (idosos) a mais os serviços quando comparados com aqueles que não relataram problema.

A Tabela 3 apresenta os resultados da análise ajustada para as três faixas etárias. Salientase que as análises foram ajustadas para a idade como variável contínua, pois as faixas etárias utilizadas apresentavam ampla variação. $\mathrm{Na}$ análise bruta, tanto quanto na ajustada, consulta foi fortemente associada com nível econômico e escolaridade (associação linear $\mathrm{p}<0,001$ ). A utilização de serviços odontológicos aumentou conforme maior nível econômico e escola- ridade, bem como melhor a autopercepção de saúde. Nos adolescentes, quanto maior o nível econômico, a escolaridade e melhor a percepção de saúde bucal, maior a utilização dos serviços. Adolescentes de nível econômicos A/B e aqueles com nove ou mais anos de estudo consultaram, aproximadamente, $60 \%$ mais do que os de nível $\mathrm{D} / \mathrm{E}$ ou com pouca escolaridade.

Adultos de nível econômico A/B consultaram $50 \%$ mais do que aqueles de nível D/E; e os com alta escolaridade ( $\geq 9$ anos), $60 \%$ mais. Os idosos com maior escolaridade e nível econômico e que referiram algum problema odontológico, no último ano, consultaram duas vezes mais do que aqueles com pior escolaridade e nível econômico e que não tiveram problemas bucais. 
Prevalência da utilização dos serviços odontológicos (\%) e razões de prevalência (RP) e intervalos de 95\% de confiança (IC95\%) da análise bruta da utilização de serviços odontológicos segundo variáveis demográficas, sócio-econômicas e de saúde bucal. Pelotas, Rio Grande do Sul, Brasil, 2006.

\begin{tabular}{|c|c|c|c|c|c|c|}
\hline \multirow[t]{3}{*}{ Variável } & \multicolumn{6}{|c|}{ Faixa etária (anos) } \\
\hline & \multicolumn{2}{|c|}{$10-19$} & \multicolumn{2}{|c|}{$20-59$} & \multicolumn{2}{|c|}{$\geq 60$} \\
\hline & $\%$ & RP (IC95\%) & $\%$ & RP (IC95\%) & $\%$ & RP (IC95\%) \\
\hline Gênero & $p=0,4$ * & $p=0,4$ * & $p<0,001 *$ & $p<0,001$ * & $p=0,6$ * & $p=0,5$ \\
\hline Masculino & 50,6 & 1,00 & 47,8 & 1,00 & 35,7 & 1,00 \\
\hline Feminino & 53,6 & $1,1(0,9-1,2)$ & 58,5 & $1,2(1,1-1,3)$ & 38,1 & $1,1(0,9-1,3)$ \\
\hline Cor da pele & $p=0,004$ * & $p=0,04$ * & $\mathrm{p}=0,001 \star$ & $p=0,01 *$ & $p=0,4$ * & $\mathrm{p}=0,4$ * \\
\hline Branca e amarela & 54,8 & $1,3(1,0-1,6)$ & 55,3 & $1,2(1,0-1,4)$ & 37,9 & $1,2(0,8-1,6)$ \\
\hline Preta, parda e indígena & 42,9 & 1,00 & 46,5 & 1,00 & 33,0 & 1,00 \\
\hline Nível econômico ** & $p<0,001 * \star \star$ & $\mathrm{p}<0,001 * \star \star$ & $\mathrm{p}<0,001 \star \star \star$ & $p<0,001 * \star \star$ & $p<0,001 * \star \star$ & $\mathrm{p}<0,001$ *** \\
\hline D-E & 37,2 & 1,00 & 37,8 & 1,00 & 21,4 & 1,00 \\
\hline C & 54,3 & $1,5(1,2-1,8)$ & 49,6 & $1,3(1,2-1,5)$ & 31,2 & $1,5(1,0-2,1)$ \\
\hline$A-B$ & 70,4 & $1,9(1,6-2,3)$ & 71,5 & $1,9(1,7-2,1)$ & 61,1 & $2,9(2,1-4,0)$ \\
\hline Escolaridade (anos) & $p<0,001$ *** & $\mathrm{p}<0,001$ *** & 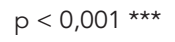 & 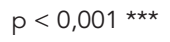 & $p<0,001$ *** & $p<0,001$ *** \\
\hline $0-4$ & 39,4 & 1,00 & 35,0 & 1,00 & 25,5 & 1,00 \\
\hline $5-8$ & 56,5 & $1,4(1,2-1,7)$ & 45,4 & $1,3(1,1-1,5)$ & 39,2 & $1,5(1,1-2,1)$ \\
\hline$\geq 9$ & 66,9 & $1,7(1,4-2,0)$ & 68,2 & $1,9(1,7-2,2)$ & 66,7 & $2,6(2,0-3,4)$ \\
\hline Situação conjugal & $p=0,34$ * & $p=0,5$ * & $p=0,01$ * & $p=0,01$ * & $p=0,5$ * & $p=0,5$ * \\
\hline Com companheiro(a) & 44,4 & 1,00 & 51,7 & 1,00 & 38,3 & 1,00 \\
\hline Sem companheiro(a) & 52,5 & $1,2(0,8-1,8)$ & 57,1 & $1,1(1,0-1,2)$ & 35,7 & $0,93(0,7-1,2)$ \\
\hline Problema odontológico & $\mathrm{p}<0,001$ * & $\mathrm{p}<0,001$ * & $\mathrm{p}<0,001 *$ & $\mathrm{p}<0,001 *$ & $\mathrm{p}<0,001$ * & $p<0,001 *$ \\
\hline Não & 42,2 & 1,00 & 42,8 & 1,00 & 26,1 & 1,00 \\
\hline Sim & 58,3 & $1,4(1,2-1,6)$ & 61,0 & $1,4(1,3-1,6)$ & 62,8 & $2,4(2,0-2,9)$ \\
\hline Autopercepção de saúde bucal & $p=0,002 * \star \star *$ & $p=0,005^{* * *}$ & 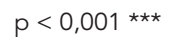 & 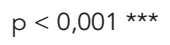 & $p=0,4$ * & $p=0,2$ * \\
\hline Ruim/Muito ruim & 47,4 & 1,00 & 40,8 & 1,00 & 46,8 & 1,00 \\
\hline Regular & 42,8 & $0,9(0,6-1,4)$ & 48,0 & $1,2(1,0-1,4)$ & 37,0 & $0,8(0,5-1,2)$ \\
\hline Boa/Muito boa & 56,1 & $1,2(0,8-1,7)$ & 59,0 & $1,4(1,2-1,7)$ & 36,1 & $0,8(0,5-1,1)$ \\
\hline Total & $52,2(447)$ & - & $53,6(1.362)$ & - & $37,2(222)$ & - \\
\hline
\end{tabular}

* Teste de Wald para heterogeneidade;

** Classificação econômica da Associação Brasileira de Empresas de Pesquisa 16;

$\star \star \star$ Teste de Wald para tendência linear.

\section{Discussão}

Em Pelotas, a prevalência de utilização de serviços odontológicos para a população de 10 anos de idade ou mais foi de 50,9\% no último ano. Entre os adolescentes, $52,2 \%$ consultaram, e, entre os adultos e idosos, o percentual foi de $53,6 \%$ e $37,2 \%$, respectivamente.

Do total de adolescentes de 10-19 anos, $24,88 \%$ do grupo de $10-14$ anos relataram nunca ter consultado o dentista; já entre os adolescentes de 15-19 anos, esse número baixou para 9,9\%. Em contraste, o estudo epidemiológico nacional de Saúde Bucal - projeto SB Brasil -, realizado em 2002-2003 6, evidenciou que apenas cerca de 6,0\% dos adolescentes de 15-19 anos nunca foram ao dentista na macrorregião Sul. Pode ter ocorrido de os adolescentes de 10-14 anos não se lembrarem de terem ido ao dentista. Outra hipótese seria de que, ao contrário dos adultos ou idosos, os adolescentes não procuram ajuda sozinhos - eles confiam nos pais, professores e nos outros adultos que os acompanham para que estes sejam capazes de identificar os sinais e sintomas e os encaminhem ao atendimento. Dessa forma, pode ser mais difícil para eles reconhecer um problema odontológico.

Quando se analisa a proporção de indivíduos que nunca utilizaram serviços odontológicos entre adultos e idosos, os dados da Região Sul do estudo nacional são semelhantes aos do presente estudo.

Alguns estudos sugerem que há maior proporção de mulheres do que de homens em idade 
Razões de prevalência (RP) e intervalo de 95\% de confiança (IC95\%) da análise ajustada de utilização de serviços odontológicos conforme variáveis demográficas, sócio-econômicas e de saúde bucal. Pelotas, Rio Grande do Sul, Brasil, 2006.

\begin{tabular}{|c|c|c|c|}
\hline \multirow[t]{2}{*}{ Variável } & \multicolumn{3}{|c|}{ Faixa etária (anos) } \\
\hline & 10-19 RP * (IC95\%) & $20-59$ RP * (IC95\%) & $\geq 60 \mathrm{RP} *(\mathrm{IC} 95 \%)$ \\
\hline \multicolumn{4}{|l|}{ Nível 1} \\
\hline Gênero & $p=0,4$ ** & $p<0,001$ ** & $p=0,2 * \star$ \\
\hline Masculino & 1,00 & 1,00 & 1,00 \\
\hline Feminino & $1,1(0,9 ; 1,2)$ & $1,2(1,1-1,3)$ & $1,1(0,9-1,4)$ \\
\hline Cor da pele & $p=0,7 \star \star$ & $p=0,7^{\star \star}$ & $p=0,8 * \star$ \\
\hline Branca e amarela & $1,0(0,8-1,3)$ & $1,0(0,9-1,1)$ & $1,0(0,7-1,5)$ \\
\hline Preta, parda e indígena & 1,00 & 1,00 & 1,00 \\
\hline Nível econômico (ABEP) & $p<0,001 * \star \star$ & $p<0,001$ *** & $p=0,001 \star \star \star \star$ \\
\hline$D-E$ & 1,00 & 1,00 & 1,00 \\
\hline C & $1,3(1,1-1,6)$ & $1,1(1,0-1,3)$ & $1,2(0,8-1,7)$ \\
\hline$A-B$ & $1,6(1,3-2,0)$ & $1,5(1,3-1,7)$ & $1,8(1,2-2,6)$ \\
\hline Escolaridade (anos) & $p<0,001 * \star \star$ & $p<0,001$ *** & 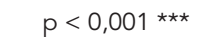 \\
\hline $0-4$ & 1,00 & 1,00 & 1,00 \\
\hline $5-8$ & $1,4(1,2-1,8)$ & $1,2(1,0-1,4)$ & $1,4(1,0-1,9)$ \\
\hline$\geq 9$ & $1,7(1,3-2,2)$ & $1,6(1,3-1,8)$ & $2,0(1,5-2,6)$ \\
\hline Situação conjugal & $p=0,6$ ** & $p=0,2$ ** & $p=0,6$ ** \\
\hline Com companheiro(a) & 1,00 & 1,00 & 1,00 \\
\hline Sem companheiro(a) & $1,1(0,8-1,7)$ & $1,1(1,0-1,1)$ & $1,1(0,9-1,3)$ \\
\hline \multicolumn{4}{|l|}{ Nível 2} \\
\hline Problema odontológico & $p<0,001$ ** & $p<0,001$ ** & $p<0,001$ ** \\
\hline Não & 1,00 & 1,00 & 1,00 \\
\hline $\operatorname{Sim}$ & $1,4(1,2-1,7)$ & $1,5(1,3-1,6)$ & $2,0(1,7-2,4)$ \\
\hline \multicolumn{4}{|l|}{ Nível 3} \\
\hline Autopercepção de saúde bucal & $p=0,005 * \star \star$ & 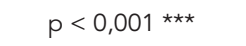 & $p=0,5^{\star \star \star}$ \\
\hline Ruim/Muito ruim & 1,00 & 1,00 & 1,00 \\
\hline Regular & $0,8(0,6-1,2)$ & $1,1(0,9-1,3)$ & $1,0(0,7-1,4)$ \\
\hline Boa/Muito boa & $1,1(0,8-1,6)$ & $1,4(1,1-1,6)$ & $1,1(0,8-1,5)$ \\
\hline
\end{tabular}

* Análise ajustada para variáveis do mesmo nível e níveis superiores e idade $(p<0,2)$;

** Teste de Wald para heterogeneidade;

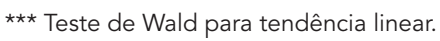

adulta que utilizaram os serviços odontológicos no ano anterior e também que elas visitam mais vezes o dentista 2,13,14. As mulheres adultas, neste estudo, consultaram $20 \%$ mais do que os homens, entretanto, contraditoriamente, apresentam uma pior percepção de sua saúde bucal. Em estudo com adolescentes de 14-18 anos de idade, as visitas foram igualmente mais freqüentes entre as mulheres (89\%) do que entre os homens (81\%) 19. Como se pode averiguar, as mulheres geralmente consultam mais em todas as faixas etárias, entretanto, após análise multivariável ajustada, essa associação desapareceu para as adolescentes e idosas. Hipoteticamente, essa diferença pode não ter sido estatisticamente significativa porque o número da amostra para esses estratos foi menor do que para as adultas, resultando em menor poder estatístico para se detectar essas diferenças.

Confirmando resultados de outros estudos, verificou-se que adultos e jovens consultaram mais do que idosos 20. Esses procuram o atendimento principalmente por motivos de prevenção (37,47\% dos adolescentes e 30,28\% dos adultos), restauração ou endodontia (36,12\% adolescentes; $45,32 \%$ adultos) e cirurgia ( $11,96 \%$ adolescentes; $17,12 \%$ adultos). Os maiores percentuais de prevenção, em contraste com outros tratamentos observados nos adolescentes, possivelmente, devem-se ao fato de que os danos causados pelas doenças bucais aumentam com a idade. Segundo Barros \& Bertoldi 2, a proporção de indivíduos 
que consulta há menos de um ano é superior nas idades de 7-19 anos e 20-49 anos.

Sabe-se que, à medida que a renda aumenta, o idoso tende a optar pela rede privada de atendimento. Entretanto, as decisões sobre onde o idoso recebe cuidados de saúde dependem das necessidades e recursos da família e não somente da situação individual do idoso. Assim, o idoso de família de baixa renda tende a ter uma menor prioridade para sua saúde do que aqueles idosos pertencentes a uma família mais abastada 20,21. Além disso, outra possível explicação para esse achado seria de que os idosos teriam poucos dentes presentes e acreditam não precisar consultar, bem como por terem maiores dificuldades em se deslocar até o local da consulta.

A variável nível econômico, como já citado na literatura 15 , mostrou, neste trabalho, uma forte associação com o padrão de uso de serviços odontológicos. Na PNAD 2003 4, 31\% da população com rendimento mensal familiar de até um salário mínimo nunca realizou uma consulta odontológica; essa proporção caiu para $3 \%$ entre aqueles com rendimentos superiores a vinte salários mínimos. Menor renda associa-se com nível educacional, valor atribuído à saúde, estilo de vida e acesso à informação sobre cuidados de saúde 21. Contudo, pesquisas têm mostrado que, quanto maior o nível de escolaridade, maior é a utilização de serviços, até mesmo quando a renda é controlada 15 . Nesse estudo, a dificuldade financeira não foi mencionada dentre as principais causas de não consulta.

Assim como o nível econômico, a variável escolaridade influencia, em grande parte, a utilização dos serviços 2,14. Estudo realizado em Minas Gerais revelou que indivíduos de maior escolaridade consultaram dez vezes mais o dentista do que os de baixa escolaridade, resultado em direção ao do presente trabalho. Interação entre renda e escolaridade não foi encontrada.

A percepção da condição bucal é um importante indicador de saúde, pois sintetiza a condição de saúde objetiva e subjetiva, os valores e as expectativas culturais. Alguns estudos sobre autopercepção demonstraram que a maioria das pessoas percebe sua condição bucal de maneira mais favorável, mesmo em condições clínicas insatisfatórias, e isso é decorrente, provavelmente, do fato de que muitas doenças detectadas no exame clínico são assintomáticas e, provavelmente, desconhecidas pelo indivíduo. O meio social igualmente é importante, porque as condições de vida e de trabalho qualificam, de forma diferenciada, a maneira pela qual as pessoas pensam, sentem e agem a respeito de sua saúde 22 .

Como esperado, as pessoas que referiram algum problema odontológico utilizaram mais os serviços odontológicos, sendo que, entre os idosos, aqueles portadores de problemas consultaram $100 \%$ mais do que os que não perceberam problemas. Sabe-se que agravos bucais podem exercer influência negativa na vida diária da maioria das pessoas, especialmente em indivíduos acima dos 60 anos, principalmente em pacientes edêntulos e aqueles dentados que não possuem atendimento odontológico regular.

É pertinente salientar que $18,8 \%$ das pessoas que não foram ao dentista relataram que não o fizeram devido a problemas nos serviços odontológicos. Percebe-se que há ainda muita disparidade no acesso e utilização de serviços odontológicos, embora, com relação ao atendimento médico, a utilização de serviços já é similar entre os diferentes grupos de renda. De fato, existe uma utilização baixa, mesmo em situações de necessidade evidente $(60,5 \%$ dos indivíduos admitiram ter problemas odontológicos). Sendo assim, é importante ressaltar que as ações para estimular o uso e melhorar o acesso são necessárias.

Nesse estudo, a autopercepção de saúde bucal foi associada a maior utilização de serviços apenas em adolescentes e adultos. Estudos a respeito de autopercepção mostram sua relação com fatores clínicos, como número de dentes cariados, perdidos ou restaurados; bem como com fatores subjetivos, como sintomas das doenças e capacidade da pessoa mastigar, falar ou sorrir sem problemas ${ }^{23}$. Ao mesmo tempo, a autopercepção é influenciada por fatores como idade, sexo, nível econômico e escolaridade. De modo geral, os indivíduos são capazes de perceber sua condição bucal com alguma precisão 24 , conquanto usando critérios diferentes do profissional. O profissional avalia a situação clínica por meio de parâmetros como a presença ou ausência de doença, enquanto que, para a população em geral, são mais significantes os sintomas e problemas sociais e funcionais advindos das doenças bucais.

O presente estudo foi realizado com uma amostra representativa da zona urbana do Município de Pelotas, fornecendo dados primários de base populacional da prevalência de utilização de serviços odontológicos, possibilitando identificar fatores a ela associados. O baixo percentual de perdas e recusas minimiza a possibilidade de viés de seleção. Utilizou-se um delineamento transversal, que apresenta vantagens quanto ao custo e ao tempo de coleta de dados. Devido ao fato de o efeito de delineamento inicial ter sido inicialmente calculado para 2,0 e, após a pesquisa, terse constatado 3,25 , calculou-se novamente o poder do estudo. Tendo em vista o grande tamanho da amostra de adultos, essa perda não foi signifi- 
cativa, entretanto, os resultados, entre adolescentes e idosos, devem ser observados com cautela.

Como o desfecho e as exposições foram coletados em um único momento, os achados podem ser afetados pelo viés de causalidade reversa, ou seja, as exposições estudadas podem ter sido influenciadas pela ocorrência do desfecho. No presente estudo, deve-se considerar a possibilidade de que a utilização de serviços odontológicos poderia modificar o comportamento de algumas variáveis explanatórias, tais como: identificação de problema odontológico no último ano e autopercepção de saúde bucal. Conseqüentemente, as associações identificadas com essas variáveis devem ser interpretadas com prudência. Outra possível limitação do estudo consiste na possibilidade de ocorrência de erro recordatório, por terem sido questionadas informações referentes a um período de 12 meses anterior ao inquérito. Outra implicação seria de haver um viés de memória diferenciado, dependendo da idade do entrevistado. É possível a hipótese de adolescentes mais jovens, por exemplo, recordarem-se, com menor freqüência, de terem utilizado os serviços odontológicos.
Indivíduos adultos do sexo masculino, de nível econômico baixo, com menos de quatro anos de escolaridade, relatando não identificar problema odontológico e com percepção de saúde bucal ruim ou muito ruim foram os que menos utilizaram os serviços odontológicos.

Recomenda-se intensificar programas educativos a fim de estimular o uso correto dos métodos preventivos aos principais agravos bucais, bem como incentivar e garantir visitas regulares aos serviços odontológicos para a realização de exames regulares desde a tenra idade, com o intuito de que os indivíduos não procurassem consultar apenas no momento em que um problema odontológico já estivesse instalado.

Para aumentar a necessária utilização de serviços odontológicos, são concorrentes medidas de caráter geral, tais como a redução das disparidades sócio-econômicas e educacionais ao lado da ampliação da oferta de serviços odontológicos no âmbito no Sistema Único de Saúde. A oferta de serviços, embora universal, deve priorizar os grupos populacionais com maiores dificuldades no acesso e utilização desses serviços.

\section{Resumo}

O objetivo deste estudo foi investigar padrões de utilização de serviços odontológicos na população de um município de porte médio. Realizou-se um inquérito de base populacional em Pelotas, Rio Grande do Sul, Brasil, com uma amostra de 4.226 indivíduos de dez anos ou mais. Empregou-se a regressão de Poisson para analisar os fatores associados; 50,9\% (IC95\%: 49,3$52,4)$ dos entrevistados consultaram com dentista no último ano. A proporção da população que realizou consultas, no último ano, entre adolescentes, adultos e idosos, foi de 52,2\%, 53,6\% e 37,2\%, respectivamente. Após ajuste para fatores de confusão, os determinantes da utilização de serviços para essas três faixas etárias foram: escolaridade $\geq 9$ anos, maior nível econômico e auto-referência à existência de problemas bucais. Ademais, adolescentes e adultos que avaliaram sua saúde bucal como boa apresentaram uma prevalência de utilização $10 \%$ e 40\% maiores em relação àqueles que avaliaram sua saúde bucal como ruim. A determinação da prevalência de utilização dos serviços odontológicos e das características de seus usuários pode auxiliar no planejamento de futuras ações em saúde que priorizem grupos populacionais com maiores dificuldades na utilização desses serviços.

Serviços de Saúde Bucal; Assistência Odontológica; Saúde Bucal 


\section{Colaboradores}

C. S. Araújo participou de todas as fases de produção do estudo, desde o planejamento até a coleta e análise dos dados e redigiu o artigo. R. C. Lima, M. A. Peres e A. J. D. Barros contribuíram no planejamento, análise estatística, interpretação dos achados e na redação do artigo.

\section{Agradecimentos}

Camila Silveira de Araújo agradece à Coordenação de Aperfeiçoamento de Pessoal de Nível Superior (CAPES) pelo apoio financeiro. Este artigo é baseado na dissertação de mestrado de Camila Silveira de Araújo, intitulada Utilização de Serviços Odontológicos no Último Ano pela População de Pelotas: Um Estudo de Base Populacional, realizada no Programa de Pós-graduação em Epidemiologia, Universidade Federal de Pelotas (UFPel).

\section{Referências}

1. Matos DL, Giatti L, Lima-Costa MF. Fatores sócio-demográficos associados ao uso de serviços odontológicos entre idosos brasileiros: um estudo baseado na Pesquisa Nacional por Amostra de Domicílios. Cad Saúde Pública 2004; 20:1290-7.

2. Barros AJD, Bertoldi AD. Desigualdades na utilização e no acesso a serviços odontológicos: uma avaliação em nível nacional. Ciênc Saúde Coletiva 2002; 7:709-17.

3. Instituto Brasileiro de Geografia e Estatística. Pesquisa Nacional por Amostra de Domicílios (PNAD 98). Rio de Janeiro: Fundação Instituto Brasileiro de Geografia e Estatística; 1998.

4. Fernandes LS, Peres MA. Association between primary dental care and municipal socioeconomic indicators. Rev Saúde Pública 2005; 39:930-6.

5. Instituto Brasileiro de Geografia e Estatística. Pesquisa Nacional de Amostra de Domicílios 2003: microdados. Rio de Janeiro: Instituto Brasileiro de Geografia e Estatística; 2004.

6. Ministério da Saúde. Projeto SB Brasil 2003: condições de saúde bucal da população brasileira 2002 2003: resultados principais. Brasília: Ministério da Saúde; 2004.

7. Sheiham A. Impact of dental treatment on the incidence of dental caries in children and adults. Community Dent Oral Epidemiol 1997; 25:104-12.

8. Pinto VG. Relacionamento entre padrões de doença e serviços de atenção odontológica. Rev Saúde Pública 1989; 23:509-14.

9. Grytten J, Holst D. Do young adults demand more dental services as their income increases? Community Dent Oral Epidemiol 2002; 30:463-9.

10. Grytten J, Rongen G. Efficiency in provision of public dental services in Norway. Community Dent Oral Epidemiol 2000; 28:170-6.

11. Grytten J, Rongen G, Asmyhr O. Subsidized dental care for young men: its impact on utilization and dental health. Health Econ 1996; 5:119-28.

12. Grytten J, Lund E, Rongen G. Equity in access to public dental services: the experience from Norway. Acta Odontol Scand 2001; 59:372-8.

13. Nguyen L, Hakkinen U, Rosenqvist G. Determinants of dental service utilization among adults - the case of Finland. Health Care Manag Sci 2005; 8:335-45.
14. Moreira RS, Nico LS, Tomita NE, Ruiz T. A saúde bucal do idoso brasileiro: revisão sistemática sobre o quadro epidemiológico e acesso aos serviços de saúde bucal. Cad Saúde Pública 2005; 21:1665-75.

15. Matos DL, Lima-Costa MF, Guerra HL, Marcenes W. Projeto Bambuí: estudo de base populacional dos fatores associados com o uso regular de serviços odontológicos em adultos. Cad Saúde Pública 2001; 17:661-8.

16. Associação Brasileira de Empresas de Pesquisa. Critério de classificação econômica Brasil 2006. http://www.abep.org/codigosguias/ABEP_CCEB. pdf. In (acessado em 31/Out/2006).

17. Rouquaryol MZ, Almeida Filho N. Epidemiologia e saúde. 6a Ed. Rio de Janeiro: Editora MEDSI; 2003.

18. Barros AJ, Hirakata VN. Alternatives for logistic regression in cross-sectional studies: an empirical comparison of models that directly estimate the prevalence ratio. BMC Med Res Methodol 2003; 3:21.

19. Honkala E, Reimpela A, Rimpela M, Jokela J. Dental services between 1977 and 1995 by finnish adolescents of different socioeconomic levels. Community Dent Oral Epidemiol 1997; 25:385-90.

20. Bos AM, Bos AJ. Determinantes na escolha entre atendimento de saúde privada e pública por idosos. Rev Saúde Pública 2004; 38:113-20.

21. Lucas SD, Portela MC, Mendonca LL. Variações no nível de cárie dentária entre crianças de 5 e 12 anos em Minas Gerais, Brasil. Cad Saúde Pública 2005; 21:55-63.

22. Silva SR, Castellanos-Fernandes RA. Autopercepção das condições de saúde bucal por idosos. Rev Saúde Pública 2001; 35:349-55.

23. Slade GD, Spencer AJ. Social impact of oral conditions among older adults. Aust Dent J 1994; 39: 358-64.

24. Reisine ST, Bailit HL. Clinical oral health status and adult perceptions of oral health. Soc Sci Med [Med Psychol Med Sociol] 1980; 14A:597-605.

\section{Recebido em 02/Abr/2008}

Versão final reapresentada em 16/Dez/2008 Aprovado em 14/Jan/2009 